\title{
A New Two-Step Robust Surface Matching Approach for Three-Dimensional Georeferencing of Historical Digital Elevation Models
}

\author{
Fernando J. Aguilar, Manuel A. Aguilar, Ismael Fernández, Joao G. Negreiros, Jorge Delgado, and José L. Pérez
}

\begin{abstract}
In this letter, a new approach based on a two-step (coarse and fine) automatic surface matching for registering two overlapping multidate digital elevation models (DEMs) is proposed to avoid the costly and time-consuming groundcontrol-point acquisition. The proposed methodology was tested to georeference a historical grid DEM obtained from a photogrammetric flight taken in 1977 and located at a heavily developed coastal area of Almería (southeast Spain). The reference DEM consisted of a newer DEM produced by the Andalusia Regional Government from a photogrammetric flight taken in 2001. The results obtained from this work may be deemed as very promising, showing high efficiency and accuracy for historical-DEM georeferencing. The vertical accuracy of the finally coregistered DEM was computed over a recent LiDAR-derived DEM (validation data set) which presented relatively unaltered areas, yielding a standard deviation that is fairly similar to the estimated uncertainty of the reference DEM.
\end{abstract}

Index Terms-Automatic digital elevation model (DEM) coregistration, change detection, surface matching, terrain mapping.

\section{INTRODUCTION}

$\mathbf{S}$ PATIAL registration of multidate data is required for many applications in remote sensing, such as change detection, construction of image mosaics, digital elevation model (DEM) generation from stereopairs, and orthorectification.

Taking into account that the accuracy of DEMs is clearly bound to the accuracy of the derived variables via error propagation [1], it is crucial to start from the best possible DEM, both for newly made DEMs and for historical DEMs. In the case of terrain change detection from multidate stereophotogrammetric flights, it is necessary to count on a precise and well-distributed set of ground control points (GCPs) to attain the georeferenced DEM. However, those GCPs are cumbersome to obtain in remote areas or from relatively old flights [2], simply because historical features are difficult to be currently localized, mea-

Manuscript received September 15, 2011; accepted November 8, 2011. Date of publication January 23, 2012; date of current version May 7, 2012. This work was supported by the Andalusia Regional Government, Spain, through the Excellence Research Project RNM-3575. This is a research project co-financed by the European Union through the program European Regional Development Fund.

F. J. Aguilar, M. A. Aguilar, and I. Fernández are with the Department of Agricultural Engineering, University of Almería, 04120 Almería, Spain.

J. G. Negreiros is with the Instituto Superior de Estatística e Gestão de Informação, University Nova de Lisboa, 1070-312 Lisbon, Portugal.

J. Delgado and J. L. Pérez are with the Department of Cartographic Engineering, Geodesy and Photogrammetry, University of Jaén, 23071 Jaén, Spain.

Color versions of one or more of the figures in this paper are available online at http://ieeexplore.ieee.org.

Digital Object Identifier 10.1109/LGRS.2011.2175899 sured, and even pointed out onto the digital images, depending on their scale, resolution, and radiometric quality. In addition, the current process of manual GCP measurement may be prohibitively labor intensive for large projects under operational conditions, and it does not enforce subpixel level correlation between images due to the limitation of human visual interpretation. Finally, and with regard to extracting high-quality topographic data from historical imagery, GCPs should also be of high quality and well distributed over the photographs. This is particularly important when camera calibration information is incomplete or unavailable [2], which is very common when working on historical flights.

On the other hand, the geometric correction for spatial registration of multidate data must be accurate enough, because misalignments of features at the same location could render useless results. In this sense, many researchers have adopted 3-D surface matching techniques without control points to automatically coregister multitemporal DEMs, usually using the newer DEM as the reference surface to achieve the $3-\mathrm{D}$ registration of an older and generally less accurate DEM (e.g., [3]-[9]). Registration methods using a control surface are justified particularly in areas where permanent control markers are not possible. Regarding surface matching, it is based on a 3-D conformal transformation, requiring the computation of three rotations, three translations, and a global scale parameter, so that the unoriented DEM is transformed to the coordinate system of the reference DEM [10].

Given the aforementioned shortcomings of historical-DEM coregistration, the main goal of this work is to develop, test, and validate an efficient and robust surface matching procedure, which is nonsensitive to actually true terrain changes and able to georeference historical DEMs using a newer DEM as the reference surface without the need of GCPs. In this sense, this letter is structured as follows. In Section II, the skeleton and workflow corresponding to the new surface matching approach are presented. The data sets are subjects of Section III. In Section IV, the results are presented and discussed, followed by the conclusions in Section V.

\section{Surface Matching ApproACH}

Notice that, although the procedure described along this letter has been focused on the registration of DEMs coming from the application of stereo image matching techniques over historical photography, it could also be applied to DEMs obtained from any other methodology (light detection and ranging (LiDAR), interferometric synthetic aperture radar, etc.). 


\section{A. SRIM Stage (Step 1)}

Within this stage, a 2-D shaded relief is generated for both the historical and the reference DEM. In this regard, different shaded reliefs may be tested, only changing the solar elevation and azimuth to optimize the final 3-D matching between the historical and reference DEMs. In this sense, the algorithm could be repeated until the best solution is obtained.

An automatic image matching algorithm, based on the scaleinvariant feature transform (SIFT), has been implemented to identify conjugated points in image space belonging to the reference- and historical-DEM shaded-relief images. This algorithm is able to extract features by transforming image data into scale-invariant coordinates relative to local features which turn out to be invariant to image scale and rotation. Moreover, these features are shown to provide robust matching across a substantial range of affine distortion, change in 3-D viewpoint, noise addition, and illumination change, so it can be deemed as very suitable to our practical application. Once a large data set of stable key points or features has been extracted for each image, the feature recognition algorithm proceeds by matching individual features to a database of features from known objects using a fast nearest neighbor algorithm, followed by a Hough transform to identify clusters belonging to a single object, and finally performing verification through least squares solution for consistent pose parameters. The reader may find a more indepth description of SIFT method in [11].

Once the pixel coordinates for every pair of conjugated points have been computed, the corresponding 3-D coordinates are automatically extracted from the reference shaded relief (UTM ETRS89 east and north) and reference DEM (bilinearly interpolated heights above GRS80 ellipsoid in our case). Those pairs of 3-D points, previously transformed to geocentric coordinates, allow computing an iterative least squares registration between both DEMs by means of a 3-D conformal transformation

$$
\left[\begin{array}{l}
X \\
Y \\
Z
\end{array}\right]=\lambda\left[\begin{array}{lll}
a_{11} & a_{12} & a_{13} \\
a_{21} & a_{22} & a_{23} \\
a_{31} & a_{32} & a_{33}
\end{array}\right]\left[\begin{array}{l}
x \\
y \\
z
\end{array}\right]=\left[\begin{array}{c}
\Delta X \\
\Delta Y \\
\Delta Z
\end{array}\right]
$$

where the orthonormal rotation matrix is represented by $3 \times$ 3 elements which are trigonometric functions of the rotation angles $\Omega, \Phi$, and $K$. On the other hand, $X, Y$, and $Z$ are the transformed coordinates regarding the reference system (reference DEM), with $x, y$, and $z$ being the original coordinates for the DEM to be georeferenced. Equally, $\Delta X, \Delta Y$, and $\Delta Z$ are the corresponding three translations, and $\lambda$ represents a global scale parameter.

It is noteworthy that, in each iteration, those gross errors computed between the geocentric coordinates of the conjugated points after applying the 3-D transformation were discarded and not taken into account in the next one by establishing a threshold value to avoid possible outliers due to both landscape changes and false matching points. Based on our experience, that threshold should be set up close to ten times the estimated uncertainty of the reference DEM. Anyway, it must be taken into account that the main goal of this first step is only to achieve a coarse historical-DEM orientation to be refined during the second step. Therefore, relatively large errors at this first stage can be deemed as bearable.
Finally, and by estimating the final seven transformation parameters, the computed 3-D transformation is applied to the historical DEM to obtain its approximate registration. All this process making up the basis framework was programmed using MATLAB.

\section{B. RSM Stage (Step 2)}

This second step consists of using the aforementioned reference DEM as a reference topographic surface to robustly register the historical DEM previously oriented by means of shaded-relief image matching (SRIM; step 1). Thus, this second stage may be considered as a refinement process to improve, whether it is possible, the georeferentiation results achieved through the first step.

In this way, the initial conjugated points are extracted by overlapping both DEMs and using bilinear interpolation over the reference DEM to obtain two different and planimetrically corresponding elevations. At this time, those nonoverlapping DEM points have to be detected and excluded from the matching process from here onward (i.e., each observation is assigned a zero weight). In this way, a very dense data set of $z$-differences $\left(d z_{i}\right)$ or surface residuals between historical and reference DEMs for every grid point can be computed. This set of $z$-differences arranged according to their plane position has been previously named as differential model [12].

The widely known $K$-means clustering method (e.g., [13]) is employed to take into account the potential divergences between new and old DEM elevations due to true terrain changes. Those true changes are considered here as outliers, and thus, they should be excluded from the surface matching process. The problem is to separate the $N$ absolute $z$-differences into $K$ clusters by means of an iterative algorithm that minimizes the sum of distances from each object to its cluster centroid over the remaining clusters. The result is a set of $K$ clusters that are as compact and well separated as possible.

Based on some trials (unpresented data), $K$ was recommended to take a value of four. In this way, once the four absolute $z$-difference clusters are computed, the two clusters presenting the highest mean absolute $z$-differences are to be considered as potentially revised areas and thus discarded so as not to be applied in the subsequent surface matching process. The remaining two clusters are considered as potentially matching areas where the RSM can be applied using the $M$-estimator called Tukey's biweight (TB). The TB function is one of the most commonly utilized $M$-estimators and, as noted in [14], is difficult to surpass in terms of delivering good performance in most situations. The weight function is defined as follows:

$$
w\left(u_{i}\right)= \begin{cases}\left(1-u_{i}\right)^{2}, & \text { if }\left|u_{i}\right| \leq 1 \\ 0, & \text { if }\left|u_{i}\right|>1\end{cases}
$$

$u_{i}$ denotes the standardized least squares residuals $\left(d z_{i} / \sigma\right)$, where $\sigma$ represents the standard deviation of all the $z$-differences potentially selected to be involved in the surface matching process. In this way, the diagonal weight matrix, regarding the TB function $\left[w\left(u_{i}\right)\right.$ in (2)], is introduced in an iterative and massive least squares weighted solution (e.g., [5] and [15]) to compute the so-called Molodensky-Badekas 3-D conformal transformation working over geocentric coordinates. 
TABLE I

Translations, Rotations, and Scale Changes Applied to The PREORIENTED 1977 DEM TO PRODUCE THREE DIFFERENT VERSIONS OF SYNTHETIC DEFORMATIONS

\begin{tabular}{cccc}
\hline Parameters & Version 1 & Version 2 & Version 3 \\
\hline$\Delta \mathrm{X}$ & $10 \mathrm{~m}$ & $50 \mathrm{~m}$ & $100 \mathrm{~m}$ \\
$\Delta \mathrm{Y}$ & $10 \mathrm{~m}$ & $50 \mathrm{~m}$ & $100 \mathrm{~m}$ \\
$\Delta \mathrm{Z}$ & $10 \mathrm{~m}$ & $50 \mathrm{~m}$ & $100 \mathrm{~m}$ \\
$\Delta \Omega$ & $10^{\circ}$ & $30^{\circ}$ & $45^{\circ}$ \\
$\Delta \Phi$ & $10^{\circ}$ & $30^{\circ}$ & $45^{\circ}$ \\
$\Delta \mathrm{K}$ & $10^{\circ}$ & $30^{\circ}$ & $45^{\circ}$ \\
$\Delta \lambda$ & 0.9 & 0.7 & 0.5 \\
\hline
\end{tabular}

After the estimation of the seven transformation parameters, the resulting 3-D transformation was applied to the historical DEM to refine its previous georeferentation coming from step 1 . This second stage procedure was also programmed with MATLAB.

\section{DATA SETS}

\section{A. Historical Data Set Corresponding to 1977 (DEM to Georeference)}

The previously discussed two-step RSM method was tested onto one stereopair belonging to a B\&W historical photogrammetric flight taken in 1977. This flight presented an approximate scale of $1: 18000$ and a focal distance close to $152.77 \mathrm{~mm}$. The study area comprised a heavily developed coastal area of Almería (southeast Spain).

A $10-\mathrm{m}$ grid-spacing DEM was extracted by means of stereo matching techniques (ISAE 4.0 from Z/I Imaging), ranking over previously digitized images $(15 \mu \mathrm{m} /$ pixel $\approx 30$-cm ground sample distance) with a radiometric resolution of 8 bits.

To test the capability of the developed method to deal with highly deformed DEMs (i.e., badly preoriented), different rotations, translations, and scale changes were applied to the original preoriented DEM to obtain three synthetic deformed DEMs (Table I).

\section{B. Reference Data Set Corresponding to 2001}

The reference DEM corresponding to 2001 consisted of a 10-m grid-spacing DEM produced by the Andalusia Regional Government (Spain) throughout a photogrammetric flight taken in 2001 (scale $\approx 1: 20000$ ). It has been chosen because of its quality and seamlessness, systematically covering the whole of the Andalusia region. The corresponding DEM accuracy was estimated with 62 differentially corrected Global Positioning System checkpoints located at open terrain, yielding a mean vertical error of $0.88 \mathrm{~m}$ (underestimated elevations) and a standard deviation of $1.03 \mathrm{~m}$.

\section{Validation Data Set}

The validation data set consisted of a LiDAR-derived DEM taken during August and September 2004 by means of an Optech ALTM 3025 LiDAR sensor. Among its main operational parameters, we highlighted the following ones: a flight height of $2300 \mathrm{~m}$, a point density of around 1 point $/ \mathrm{m}^{2}$, and a computed vertical accuracy between 6 and $15 \mathrm{~cm}$, depending on the land cover. The accurate and high-resolution (1-m gridspacing) raw digital surface model was filtered and decimated using TerraScan software to produce a more suitably handled 3-m grid-spacing DEM within the working area, comprising a nonurbanized and, thus, relatively stable area along a dryravine bed.

\section{RESUlts AND Discussion}

\section{A. Initial Position of the 1977 Historical DEM}

The initial position of the 1977 historical DEM presented a clearly diagonal-rotated northwest-to-southeast direction leaning as compared with the 2001 reference DEM. It produced a sparse histogram of signed vertical residuals.

The mean error took a value of $16.18 \mathrm{~m}$ (Table III), indicating a notable overall bias or systematic error. This initial misalignment could be expected given the poor coordinates of the ground points utilized to compute the absolute orientation of the historical stereopair. Meanwhile, random errors were also quite large, as can be deduced from the high standard deviation of the whole $z$-differences within the overlap area (Table III). Thus, the starting position for the historical DEM should be clearly improved. In this case, it is necessary to cope with these high local deformations by treating them as outliers while the designed algorithm, as a robust estimator technique, should be less sensitive to the existence of gross errors. It is a noneasy problem to solve because there will be coexisting matching points, gross errors (significant surface differences due to the passage of time), and boundary outliers (i.e., points within the transition area). In the remaining sections, the twostep proposed algorithm will be tested to check its ability to afford this intriguing challenge.

\section{B. $\operatorname{SRIM}($ Step 1)}

As it was previously commented, the process called SRIM makes up the first step of the proposed two-step RSM approach.

First of all, it is important to point out that the matching results may be quite variable, depending on the solar position from which the shaded-relief images were generated. In fact, an automated GCP location in two images consists of two stages. The first one extracts spatial features from each image. Then, the features are paired by correspondence matching. The success of the process depends partially on the similarity of the features in the two images, which is clearly related to the solar position. This is the reason why the proposed algorithm could be used to iteratively search for an optimal solution changing both solar azimuth and solar elevation. In our experiment, one of the best tested configurations turned out to be the case of $45^{\circ}$ solar azimuth and $45^{\circ}$ solar elevation, in which 22 conjugated points were successfully matched out of 2784 and 1722 key points found in 1977 and 2001 shaded-relief images, respectively. It is worth noting that, although there are a large number of extracted key points, the number of final matched points between both shade reliefs is quite low. It was due to the selection of the distance ratio parameter in the fast nearest neighbor algorithm developed in [11] to recognize correctly matched features. In fact, all matches in which the distance ratio (a measure of dissimilarity among features) was greater than 0.6 were rejected in order to assure the creation of a small, but reliable, set of matched points. At this point, it is imperative to state that the most important matter is not only the number of pairs achieved but also the matching precision and even distribution of those points on the working area. 
TABLE II

Estimated PARAmeters AND ACCURACIES For THE COMPUTEd 3-D CONFORMAL TRANSFORMATION (GEOCENTRIC COORDINATES With REGARD TO GRS80 REFERENCE ELLIPSOID). SRIM BASED ON $45^{\circ}$ SOlAR AZIMUTH AND $45^{\circ}$ SOLAR ElEVATION

\begin{tabular}{ccc}
\hline Parameter & Value & Accuracy \\
\hline$\Delta \mathrm{X}$ & $-30.10 \mathrm{~m}$ & $0.24 \mathrm{~m}$ \\
$\Delta \mathrm{Y}$ & $-7.78 \mathrm{~m}$ & $0.25 \mathrm{~m}$ \\
$\Delta \mathrm{Z}$ & $-21.64 \mathrm{~m}$ & $0.24 \mathrm{~m}$ \\
$\Delta \Omega$ & $0.454^{\circ}$ & $0.072^{\circ}$ \\
$\Delta \Phi$ & $0.990^{\circ}$ & $0.028^{\circ}$ \\
$\Delta \mathrm{K}$ & $-0.856^{\circ}$ & $0.092^{\circ}$ \\
$\lambda$ & 1.00007 & 0.0003 \\
\hline
\end{tabular}

TABLE III

Signed $z$-DifFerence STATISTICS Within THE OVERLAP AREA CORRESPONDING TO THE COMPARISON BETWEEN THE 1977 HISTORICAL DEM AND SEVERAL REFERENCE DEMs ( $45^{\circ}$ Solar AZIMUth AND ELEVATION WERE APPLIED TO THE SRIM ORIENTATION)

\begin{tabular}{ccccc}
\hline & \multicolumn{4}{c}{ Signed z-differences statistics (m) } \\
\cline { 2 - 5 } & Mean & $\begin{array}{c}\text { Standard } \\
\text { deviation }\end{array}$ & Maximum & Minimum \\
\hline 1977 Initial DEM - 2001 reference & 16.18 & 22.12 & 63.34 & -35.02 \\
$\begin{array}{c}\text { DEM } \\
\text { 1977 only SRIM oriented DEM - }\end{array}$ & -0.14 & 2.03 & 10.14 & -16.28 \\
$\begin{array}{c}\text { 2001 reference DEM } \\
\text { 1977 SRIM+RSM oriented DEM - }\end{array}$ & -0.20 & 1.95 & 9.38 & -15.57 \\
$\begin{array}{c}\text { 2001 reference DEM } \\
\text { 1977 only SRIM oriented DEM - } \\
\text { 2004 LiDAR-derived DEM }\end{array}$ & -0.58 & 1.27 & 3.63 & -6.00 \\
$\begin{array}{c}\text { 1977 SRIM+RSM oriented DEM } \\
\text { - 2004 LiDAR-derived DEM }\end{array}$ & $\mathbf{- 0 . 5 5}$ & $\mathbf{1 . 0 9}$ & $\mathbf{3 . 3 2}$ & $\mathbf{- 7 . 3 1}$ \\
$\begin{array}{c}\text { 2001 reference DEM - 2004 } \\
\text { LiDAR-derived DEM }\end{array}$ & $\mathbf{- 0 . 5 0}$ & $\mathbf{0 . 9 3}$ & $\mathbf{3 . 8 7}$ & $\mathbf{- 5 . 3 7}$ \\
\hline
\end{tabular}

Table II shows the estimated transformation parameters for the iteratively computed 3-D conformal transformation. It is worth reporting that the used threshold to discard GCPs along the iterative adjustment took a value of $10 \mathrm{~m}$ since the final results could be highly dependent on this variable. In this sense, seven GCPs, out of the original 22 GCPs previously matched, were discarded during the adjustment process due to exceeding the established threshold, computing the final 3-D adjustment on the remaining 15 matched GCPs. The accuracies obtained from the least squares variance-covariance matrix turned out to be fairly good (Table II), indicating a reliable solution. Briefly, the higher the number of ground points included in the least squares adjustment, the better the achieving of an even distribution over the complete working area.

As it is depicted in Table III, the initial position of the 1977 historical DEM has been notably corrected, and the matching results have been clearly improved after applying the SRIM algorithm. It is worthy to note that the standard deviation of the 1977 SRIM oriented DEM is still almost twice the one estimated for the 2001 reference DEM $(1.03 \mathrm{~m})$. So, the final matching from the SRIM process may be considered as reasonably acceptable.

By judging the numerical data shown in Table III, the SRIM stage can be used to automatically and coarsely coregister multitemporal DEMs without GCPs. It is necessary to take into account that some of the gross errors detected may be actually considered as not an error but landscape change due to, for example, earthwork projects. In this way, the proposed methodology seems to be very robust because localized shaded- relief features utilized to compute the robust 3-D conformal transformation are usually geomorphological features that remain relatively stable over a long time.

When registering multitemporal DEMs, the most important problem is associated to the intensity of temporal deformation or change that occurred between the periods of the study. In most surface matching algorithms, the deformation area is restricted to, at most, $50 \%$ by introducing the so-called differential model and improving the classic least $z$-difference algorithm. However, this approach is rather complex and needs a previous rough coregistration or knowledge about the approximated transformation to carry it out [8]. This SRIM stage has been used as the first step to later apply the previously described RSM algorithm to refine the initial matching as much as possible.

\section{C. $R S M$ (Step 2)}

Once step 1 has been concluded, it is time to refine the computed orientation by means of the aforementioned RSM described in Section II-B. It can be noticed that the computed translations along the $X, Y$, and $Z$ axes were always less than $8 \mathrm{~cm}$, whereas the computed rotations may also be considered as very small (less than $0.05^{\circ}$ in the worst of the cases). It was an expected situation given the good coarse orientation coming from the first step. The accuracy of the estimated parameters, calculated through the dispersion matrix, was very high mainly due to the huge number of available matching points which confers important soundness and robustness to this process.

After applying this second step, the 1977 SRIM oriented DEM has been slightly refined to improve on its coregistration with respect to the 2001 reference DEM, yielding the accuracy results shown in Table III. As can be checked, the results may be deemed to be fairly similar to those obtained after applying only the SRIM first step. Regarding this issue, it is essential to point out that the RSM second step has been designed to correct, if needed, the approximation carried out through the SRIM first step. In this sense, the poorer is the SRIM performance, the better is the added value from the RSM stage.

Regarding the LiDAR-derived validation DEM, which can be supposed as a relatively unaltered area during the last decades and so reasonably free of change, the maximum and minimum errors were the lowest, while the matching accuracy worked the best (Table III). The computed uncertainty (measured as standard deviation) was almost equal to that estimated for the 2001 reference DEM. In the same way, the $z$-difference analysis with regard to the comparison between the 2001 reference DEM and the 2004 LiDAR-derived DEM showed similar results (Table III), demonstrating the great efficiency of this method to obtain excellent multidate surface registrations without costly and time-consuming surveyed ground points. Furthermore, the spatial error distribution turned out to be quite stable and evenly distributed all over the working area, indicating a good performance of the proposed matching algorithm of being able to correct the poor orientation of the original historical DEM (Fig. 1).

\section{Highly Deformed DEMS}

One of the most important problems when registering multidate DEMs is closely related to the quality of the previous preorientation issue because, somehow, a bad preorientation accentuates the problems due to the presence of local deformations. 


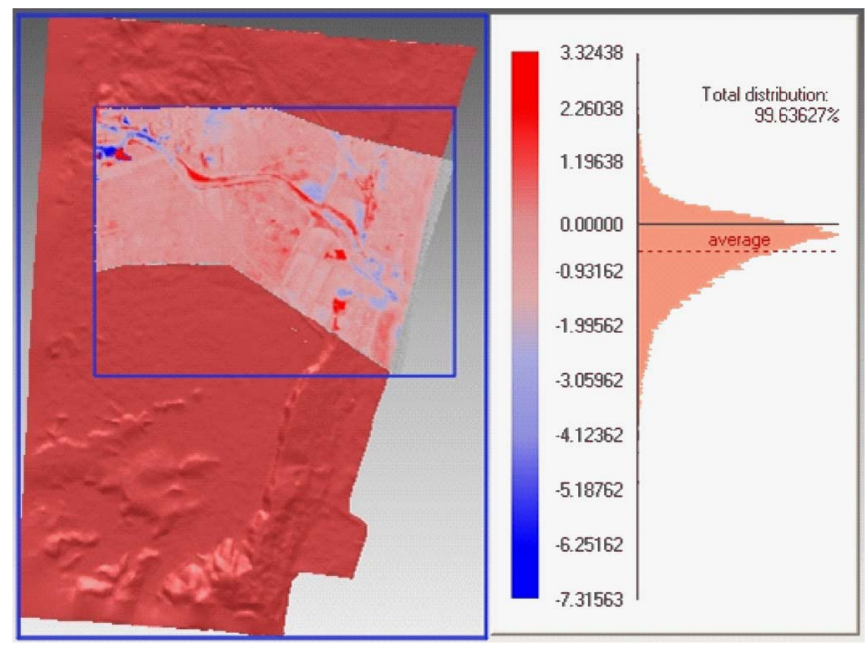

Fig. 1. Spatial distribution of signed $z$-differences (1977 SRIM + RSM oriented DEM-2004 LiDAR-derived DEM within the overlap area) and the corresponding histogram.

TABLE IV

Signed $z$-DifFERENCE STATISTICS FOR THE OVERLAP AREA CORRESPONDING TO THE COMPARISON BETWEEN DIFFERENT VERSIONS OF DEFORMED 1977 DEM AND REFERENCE DEM

\begin{tabular}{|c|c|c|c|c|}
\hline & \multicolumn{4}{|c|}{ Signed z-differences statistics (m) } \\
\hline & Mean & $\begin{array}{l}\text { Standard } \\
\text { deviation }\end{array}$ & Maximum & Minimum \\
\hline $\begin{array}{l}\text { Raw version } 11977 \text { DEM - } 2001 \\
\text { reference DEM }\end{array}$ & -0.80 & 47.03 & 80.84 & -81.48 \\
\hline $\begin{array}{l}\text { RSM oriented version } 11977 \text { DEM } \\
-2001 \text { reference DEM }\end{array}$ & -1.40 & 8.31 & 25.46 & -36.88 \\
\hline $\begin{array}{l}\text { SRIM+RSM oriented version } 1 \\
1977 \text { DEM - } 2001 \text { reference DEM }\end{array}$ & -0.94 & 1.99 & 5.68 & -19.21 \\
\hline $\begin{array}{c}\text { Raw version } 21977 \text { DEM - } 2001 \\
\text { reference DEM }\end{array}$ & 0.89 & 45.93 & 78.91 & -79.51 \\
\hline $\begin{array}{l}\text { RSM oriented version } 21977 \text { DEM } \\
-2001 \text { reference DEM }\end{array}$ & -2.78 & 23.25 & 48.56 & -49.68 \\
\hline $\begin{array}{l}\text { SRIM+RSM oriented version } 2 \\
1977 \text { DEM - } 2001 \text { reference DEM }\end{array}$ & -0.45 & 2.05 & 7.11 & -14.76 \\
\hline $\begin{array}{l}\text { Raw version } 31977 \text { DEM - } 2001 \\
\text { reference DEM }\end{array}$ & -0.10 & 30.10 & 51.59 & -52.00 \\
\hline $\begin{array}{l}\text { RSM oriented version } 31977 \text { DEM } \\
\quad-2001 \text { reference DEM }\end{array}$ & 5.94 & 15.73 & 38.13 & -28.30 \\
\hline $\begin{array}{l}\text { SRIM+RSM oriented version } 3 \\
1977 \text { DEM - } 2001 \text { reference DEM }\end{array}$ & -0.25 & 2.54 & 12.33 & -16.49 \\
\hline
\end{tabular}

Summing up, what is needed is a relatively well-preoriented historical DEM to obtain accurate results. In fact, our second step (RSM approach) was not able to fully register any type of the synthetic deformations applied to the original 1977 DEM, as it can be observed in Table IV (although it is worth noting that those deformations are not usual under operational conditions). Therefore, and for those cases, it was necessary to apply a first step process, the so-called SRIM algorithm, to get ready a well-preoriented DEM to be refined by the second step based on the RSM algorithm. In this sense, the obtained results may be believed as fairly accurate, taking into account the extreme deformations, likely far away from real data sets, applied to the original 1977 historical DEM to test the robustness and soundness of the proposed two-step methodology.

\section{CONCLUSION}

The results obtained from this work may be regarded as very promising, showing a good coregistration between reference and historical DEMs in heavily developed coastal areas.
The second step process, based on an RSM algorithm which needs a relatively good first approximation, can be applied successfully only if the preoriented historical DEM turns out not to be excessively misaligned with respect to the reference DEM. In the case of very badly preoriented DEMs, and likely also for intense temporal deformations or terrain changes, it is necessary to take into consideration a previous step headed up to correct such issues, which can be afforded by means of the proposed SRIM. In such situations, the second step method proposed in this letter could be applied as a refining method to polish subtle deficiencies coming from the first step.

Certainly, the point is the high efficiency and robustness demonstrated by our two-step surface matching approach for historical-DEM 3-D georeferencing, particularly when it is compared to the costly and time-consuming traditional methods.

\section{ACKNOWLEDGMENT}

The authors would like to thank Prof. Pedro S. Coelho (Dean of the Instituto Superior de Estatística e Gestão de Informação, University Nova de Lisboa, Lisbon, Portugal) and Prof. M. Painho for their valuable help during his fellowship at Universidade Nova de Lisboa. Most of the presented work was carried out during this fellowship.

\section{REFERENCES}

[1] F. J. Aguilar, J. P. Mills, J. Delgado, M. A. Aguilar, J. G. Negreiros, and J. L. Pérez, "Modelling vertical error in LiDAR-derived digital elevation models," ISPRS J. Photogramm. Remote Sens., vol. 65, no. 1, pp. $103-$ 110, Jan. 2010

[2] T. D. James, T. Murray, N. E. Barrand, and S. L. Barr, "Extracting photogrammetric ground control from LiDAR DEMs for change detection," Photogramm. Rec., vol. 21, no. 116, pp. 312-328, Dec. 2006.

[3] P. E. Miller, M. Kunz, J. P. Mills, M. A. King, T. Murray, T. D. James, and S. H. Marsh, "Assessment of glacier volume change using ASTER-based surface matching of historical photography," IEEE Trans. Geosci. Remote Sens., vol. 47, no. 7, pp. 1971-1979, Jul. 2009.

[4] D. Rosenholm and K. Torlegard, "Three-dimensional absolute orientation of stereo models using digital elevation models," Photogramm. Eng. Remote Sens., vol. 54, no. 10, pp. 1385-1389, 1989.

[5] L. J. Pilgrim, "Robust estimation applied to surface matching," ISPRS J. Photogramm. Remote Sens., vol. 51, no. 5, pp. 243-257, Oct. 1996.

[6] Z. Li, Z. Xu, M. Cen, and X. Ding, "Robust surface matching for automated detection of local deformations using least-median-of-squares estimator," Photogramm. Eng. Remote Sens., vol. 67, no. 11, pp. $1283-$ 1292, Nov. 2001.

[7] P. E. Miller, J. P. Mills, S. J. Edwards, P. Bryan, S. Marsh, H. L. Mitchell, and P. Hobbs, "A robust surface matching technique for coastal geohazard assessment and management," ISPRS J. Photogramm. Remote Sens., vol. 63 , no. 5 , pp. 529-542, Sep. 2008.

[8] T. Zhang, M. Cen, and G. Zhou, "Debris-flow detection along highway using multi-temporal DEMs," Int. J. Remote Sens., vol. 27, no. 21, pp. 49114921, 2006.

[9] D. Akca, "Co-registration of surfaces by 3D least squares matching," Photogramm. Eng. Remote Sens., vol. 76, no. 3, pp. 307-318, 2010.

[10] J. P. Mills, S. J. Buckley, H. L. Mitchell, P. J. Clarke, and S. J. Edwards, "A geomatics data integration technique for coastal change monitoring," Earth Surf. Process. Landforms, vol. 30, no. 6, pp. 651-664, 2005.

[11] D. G. Lowe, "Distinctive image features from scale-invariant keypoints," Int. J. Comput. Vis., vol. 60, no. 2, pp. 91-110, 2004.

[12] T. Zhang, M. Cen, X. Wu, and G. Zhou, "Fully automatic DEM deformation detection without control points using differential model based on LZD algorithm," in Proc. IGRSS, Seoul, Korea, 2005, pp. 25-29.

[13] H. Spath, Cluster Dissection and Analysis: Theory, FORTRAN, Programs, Examples. New York: Halsted, 1985.

[14] C. Goodall, " $M$-Estimators of location: An outline of the theory," in Understanding Robust and Exploratory Data Analysis, D. Hoaglin, F. Mosteller, and J. W. Tukey, Eds. New York: Wiley, 1983, pp. 339-403. [15] A. L. Allan, Maths for Map Makers. Dunbeath, U.K.: Whittles Pub., 2004. 